\title{
Impaired glucose tolerance in Fanconi-Bickel syndrome: Eight patients with two novel mutations
}

\author{
Berna Şeker-Yılmaz¹, Deniz Kör², Fatma Derya Bulut ${ }^{3}$, Bilgin Yüksel ${ }^{4}$ Aysun Karabay- \\ Bayazıt $^{5}$, Ali Kemal Topaloğlu ${ }^{4}$, Gülay Ceylaner ${ }^{6}$ Neslihan Önenli-Mungan ${ }^{3}$ \\ ${ }^{1}$ Department of Pediatric Metabolism, Mersin City Hospital, Mersin; ${ }^{2}$ Department of Pediatric Metabolism, Adana City \\ Hospital, Adana; ${ }^{3}$ Departments Pediatric Metabolism, ${ }^{4}$ Pediatric Endocrinology, ${ }^{5}$ Pediatric Nephrology, Balcali Hospital, \\ Cukurova University, Adana; ${ }^{6}$ Intergen Genetics Laboratory, Ankara, Turkey.Email: berna_seker@yahoo.co.uk \\ Received: 13th February 2017, Revised: 11th April 2017, Accepted: 23th April 2017
}

SUMMARY: Șeker-Yılmaz B, Kör D, Bulut FD, Yüksel B, Karabay-Bayazıt A, Topaloğlu AK, Ceylaner G, Önenli-Mungan N. Impaired glucose tolerance in Fanconi-Bickel syndrome: Eight patients with two novel mutations. Turk J Pediatr 2017; 59: 434-441.

Fanconi-Bickel syndrome (FBS) is a rare, autosomal recessive disorder of carbohydrate metabolism caused by defects in the facilitative glucose transporter 2 (GLUT2 or SLC2A2) gene. Prominent findings are failure to thrive, renal tubular acidosis, hypoglycemia and postprandial hyperglycemia even mimicking diabetes mellitus.

Eight patients from 6 families with FBS were included in this study. c.482_483insC homozygous mutation was detected in six patients from four different families. Mutation analysis of SLC2A2 gene revealed two novel homozygous mutations; c.1069delGinsAATAA and c.575A>G.

Standard oral glucose tolerance test with $1.75 \mathrm{~g} / \mathrm{kg}$ oral glucose was performed in six of the patients who were older than 3-years of age. Impaired glucose tolerance was found in all patients as expected and two of them had overt diabetes. None of the antidiabetic medications were given to them in order to avoid significant hypoglycemia. Beside the conservative treatment, follow up with frequent oral glucose tolerance tests are planned.

We report these cases of FBS, as GSD XI is rare, two novel mutations were detected and also to highlight the risk of diabetes mellitus; although there is not a consensus about the treatment.

Key words: Fanconi-Bickel syndrome, oral glucose tolerance test, hypoglycemia, postprandial hyperglycemia.

Fanconi-Bickel syndrome (FBS), also called glycogen storage disease type XI, has been known since the 1940s and was first described by Fanconi and Bickel. ${ }^{1}$ It is an autosomal recessive carbohydrate metabolism disorder, caused by mutations in the GLUT2 (SLC2A2) gene on chromosome 3q26.1-26.3, encoding facilitative glucose transporter protein 2 (GLUT2). ${ }^{2}$ GLUT2 is expressed in hepatocytes, pancreatic beta cells, renal tubular cells and enterocytes. ${ }^{3}$ In hepatocyte, it provides glucose release in response to the decrease of plasma glucose levels. In pancreatic beta cells, according to changes in plasma glucose levels, GLUT2 adjusts proper insulin secretion. GLUT2 also plays an important role in monosaccharide transport both in renal tubular cells and enterocytes. ${ }^{3}$ SLC2A2 mutations leads to FBS present with failure to thrive, hepatomegaly, hypophosphatemic rickets and renal Fanconi syndrome (proteinuria, glycosuria, phosphaturia and generalized aminoaciduria). Similar to some other hepatic glycogen storage diseases presenting with fasting hypoglycemia, postprandial hyperglycemia is common for this syndrome. 
Postprandial hyperglycemia is caused by the defective insulin secretion of the pancreatic beta cells and reduced glucose uptake by the liver and rarely overt diabetes develops. ${ }^{4-5}$ In animal studies SLC2A2 inactivated mice presents with significant hyperglycemia with low levels of insulin and exogenous insulin infusion improves the symptoms in these mice and prevents the mortality ${ }^{6}$. Recent studies showed that restorage of SLC2A2 expression provides normalized insulin secretion from the pancreatic beta cells. ${ }^{7}$

Although FBS is generally considered a welldefined disease, phenotypic variability including atypical phenotype, and variable clinical severity from mild presentation to overt diabetes mellitus has been reported. ${ }^{8}$

There is no specific treatment of FBS, the management for renal Fanconi syndrome includes replacement of fluid and electrolytes; supplementation of vitamin $\mathrm{D}$, calcium, phosphorus, and bicarbonate. Small frequent meals with uncooked cornstarch are recommended in order to avoid hypoglycemia. ${ }^{9}$

We tested 8 patients with FBS whether SLC2A2 gene mutations cause impaired glucose tolerance even diabetes and furthermore we determined if there is a relationship between the mutation and the phenotype.

\section{Materials and Methods}

Eight patients from six different consanguineous parents, diagnosed with FBS were included in the study. Failure to thrive, hepatomegaly, hypophosphatemic rickets and tubulopathy were the common findings. Laboratory findings are presented in Table I.

The study was reviewed and approved by an institutional review board. All of the diagnostic tests and treatment applied to the patients were part of the routine follow up. Written informed consent were provided by both of the parents.

\section{Subject Selection}

Subject 1

A 4-months-old boy was admitted for the evaluation of failure to thrive. The baby was the first issue of consanguineous Turkish parents and had been born through normal vaginal delivery at term with birth weight of $3 \mathrm{~kg}$; without any significant antenatal and postnatal history. Exclusively breastfeeding was continued.

Physical examination detected; weight $4700 \mathrm{~g}$, head circumference $37 \mathrm{~cm}$, and height $54 \mathrm{~cm}$ (all were below 3rd percentile). Anterior fontanel was wide open, craniotabes, Harrison's groove and rachitic rosaries were noticed. Abdominal examination detected hepatomegaly $(4 \mathrm{~cm})$, without any other organomegaly or ascites.

Liver transaminases were mildly elevated. Blood urea and serum creatinine, uric acid and lactate levels were within normal limits. Investigation revealed normal serum calcium, reduced phosphorus and markedly elevated serum alkaline phosphatase. Urine biochemistry showed $\mathrm{pH}$ of 6 , specific gravity 1.026 , glucosuria $2+$, proteinuria $2+$. Normal anion gap metabolic acidosis was significant. Wrist X-ray showed features of active rickets. Ultrasonography of abdomen revealed hepatomegaly with normal echotexture.

All of these features suggested a liver disease concomitant with proximal renal tubular acidosis. As galactosemia and tyrosinemia were excluded with normal bilirubin levels, also mitochondrial diseases were excluded with normal lactic acid levels and urine fructose was negative, consequently the most probable diagnosis was FBS.

\section{Subject 2 and 3}

A one-year-old girl, first child of first-degree consanguineous parents, presented with failure to thrive, recurrent vomiting and dehydration attacks. Hepatomegaly, abdominal distension and rickets were the presenting findings. The rest of the systemic examination were normal. Blood urea nitrogen, creatinine and uric acid levels were normal. Liver transaminases are mildly elevated. After the documentation of glucosuria, proteinuria and aminoaciduria indicated proximal renal tubular acidosis. Abdominal ultrasonography revealed hepatomegaly with normal echotexture. Episodes of metabolic acidosis with normal anion gap was remarkable. Fasting hypoglycemia and postprandial hyperglycemia were recorded as expected during 


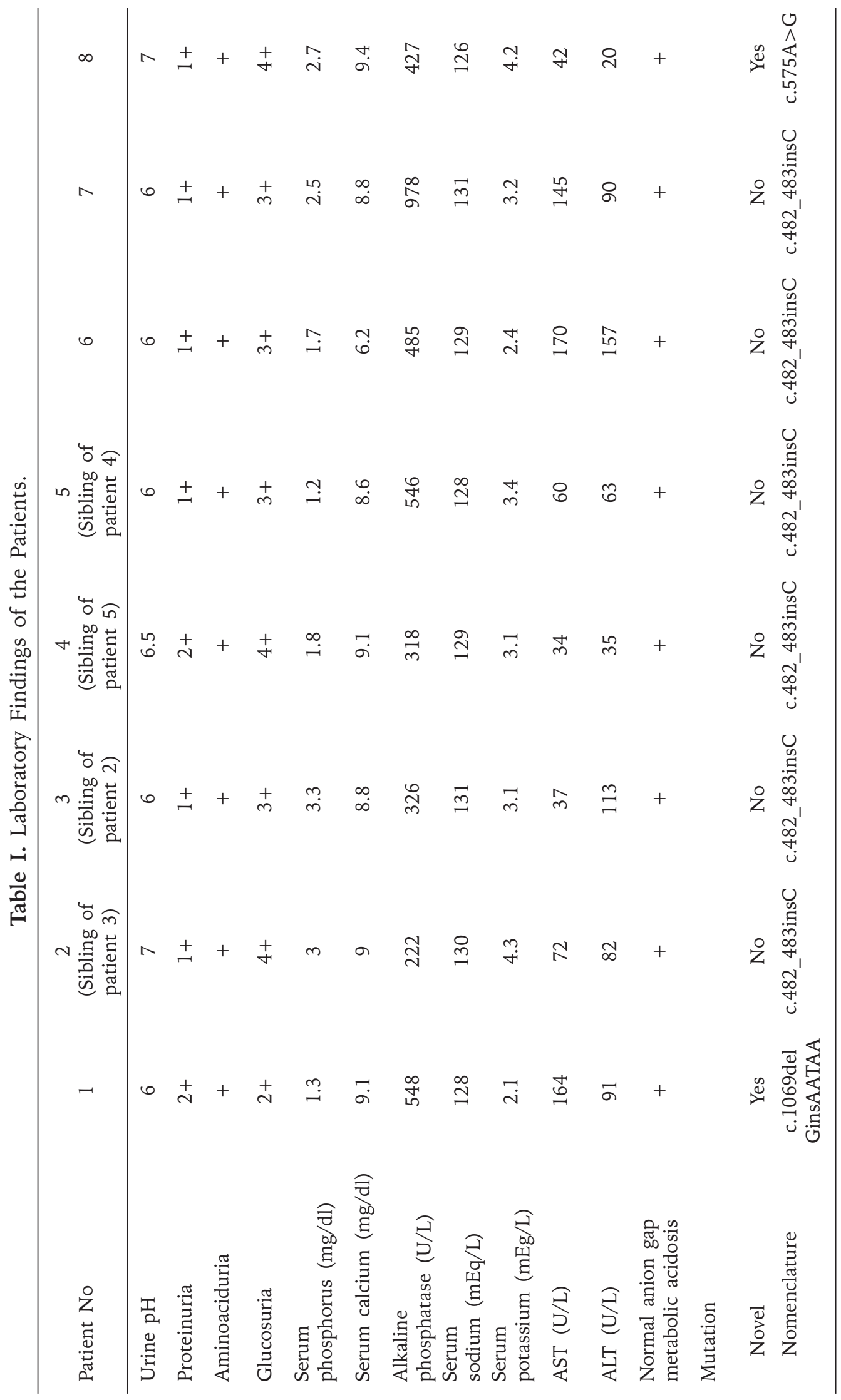


the course of the disease. Since the findings of hypophosphatemic rickets, proximal renal tubular dysfunction, episodes of normal anion gap metabolic acidosis, hepatomegaly and liver dysfunction, FBS was the probable diagnosis.

At the age of 6 years old, the parents decided to have a second child. Although genetic counselling and prenatal diagnosis were recommended, none were accepted by the family. Second child of the family was asymptomatic in the first 4 months of life, except mildly elevated transaminases. At the age of six months, symptom started to be apparent. Failure to thrive, episodes of metabolic acidosis, features of hypophosphatemic rickets, proximal renal tubulopathy, and fasting hypoglycemia appeared.

\section{Subject 4 and 5}

A 16-year-old boy, born to a third-degree consanguineous couple presented with failure to thrive and vomiting at the age of 9 months. $\mathrm{He}$ also had progressive abdominal distension since the age of 3 months. Soft hepatomegaly was observed without splenomegaly or ascites. Liver transaminases were mildly elevated without any evidence of cholestasis. After the administration, features of active rickets were noted and he was diagnosed as hypophosphatemic rickets and oral vitamin D treatment and phosphate supplementation were given. Generalized aminoaciduria, glucosuria and mild proteinuria pointed out proximal renal tubular acidosis. Normal anion gap metabolic acidosis accompanied with vomiting attacks. Since the presence of generalized proximal renal tubular acidosis, hypophosphatemic rickets, hepatomegaly and liver dysfunction, FBS was the suspected diagnosis. During the growingup period the main symptoms were fasting hypoglycemia and postprandial hyperglycemia. Then, short stature was markedly evident and puberty was delayed. Bone mineral analysis by dual energy X-ray absorptiometry revealed low bone mineral density with the lumbar vertebrae $z$ score -2.3 and intravenous bisphosphonate treatment was given.

Despite being informed, the parents of the patient did not consider prenatal diagnosis and had a second child from an unplanned pregnancy. Although the parents had detailed knowledge of the disease, they did not bring the sibling until the age of 19 months. Walking delay and abdominal distension were the complaints of admission. Doll-like face, developmental delay, hepatomegaly, and active rickets were the findings of the physical examination. Abdominal ultrasonography verified hepatomegaly. Episodes of fasting hypoglycemia was common. Proximal renal tubular acidosis was evident with the presence of generalized aminoaciduria, mild proteinuria and glucosuria. Due to the family history, FBS was the suspected diagnosis as the elder sibling and the diagnosis was confirmed by the molecular studies.

\section{Subject 6}

The patient was the first child of consanguineous Turkish parents and had been born through normal vaginal delivery without any significant prenatal and postnatal history. First symptoms of the disease, appeared when the patient was 4 months old, implicated polyuria and excessive thirst. In the following days he developed generalized proximal renal tubular defect with

Table II. Oral Glucose Tolerance Test Results.

\begin{tabular}{lcccccc}
\hline & $\begin{array}{c}\text { Fasting } \\
\text { glucose } \\
(\mathrm{mg} / \mathrm{dl}) \\
(\mathrm{N}: 70-105)\end{array}$ & $\begin{array}{c}\text { Fasting } \\
\text { insulin } \\
(\mathrm{pmol} / \mathrm{L}) \\
(\mathrm{N}:<174)\end{array}$ & $\begin{array}{c}120 \mathrm{~min} \\
\text { glucose } \\
(\mathrm{mg} / \mathrm{dl}) \\
(\mathrm{N}: 70-120)\end{array}$ & $\begin{array}{c}120 \mathrm{~min} \\
\text { insulin } \\
(\mathrm{pmol} / \mathrm{L}) \\
(\mathrm{N}: 111-1153)\end{array}$ & $\begin{array}{c}\text { Mean HbA1c } \\
(\%)\end{array}$ & $\begin{array}{c}\text { Last HbAlc } \\
(\%)\end{array}$ \\
\hline Patient 2 & 99 & 29.3 & 176 & 82.7 & 5.9 & 5.4 \\
Patient 4 & 83 & 25.4 & 266 & 8.7 & 5.6 & 5.3 \\
Patient 5 & 87 & 40.4 & 184 & 108.8 & 5.9 & 5.6 \\
Patient 6 & 43 & 2.2 & 185 & 50.9 & 5.1 & 5.3 \\
Patient 7 & 55 & 0.69 & 160 & 63.7 & 4.9 & 4.4 \\
Patient 8 & 63 & 12.5 & 252 & 268.7 & 5.8 & 6.0 \\
\hline
\end{tabular}


polyuria, aminoaciduria and glucosuria. At the age of six months abdominal distension due to the significant hepatomegaly was remarkable. Liver function tests revealed slightly elevated transaminases. According to the excessive phosphate wasting, features of hypophosphatemic rickets came out. The most probable cause was a glycogen storage disease with renal involvement. FBS was the confirmed diagnosis by the mutation analysis.

\section{Subject 7}

He was the third offspring of first degree cousins. The mother had one spontaneous abortion at 3 months of gestation. There was no significant family history. At the age of six months he was admitted to the hospital for the complaints of failure to thrive and abdominal distention. Initial physical examination revealed firm and smooth hepatomegaly with short stature. Doll-like face was also noted. Ultrasonography confirmed normal echotextured hepatomegaly. Liver enzymes were elevated. At the age of twelve months, the anterior fontanel was wide open, pectus excavatum, Harrison sulcus, widening of wrists and ankles was noticed. He was treated for hypophosphatemic rickets with sodium phosphate and vitamin D. Urine analysis revealed proteinuria and glucosuria with generalized aminoaciduria. During the follow-up, hypoglycemic episodes were noted. Glycosylated hemoglobin levels were lower than the normal range. According to these features, FBS was the suspected diagnosis.

\section{Subject 8}

A four-year-old boy, second-born of first-degree consanguineous parents, presented with failure to thrive. It was learned that his uncle had died at the age of 7 years because of liver failure. Physical examination was normal except mild hepatomegaly. Proteinuria and glucosuria were significant. Normal anion gap metabolic acidosis with hypophosphatemia indicated renal tubular acidosis. Urine aminoacid analysis showed generalized aminoaciduria. Liver transaminases were mildly elevated. Bone survey revealed the radiographic evidence of rickets with delayed bone age. Hypoglycemia was not noted and glycosylated hemoglobin levels were normal. Based on these findings FBS was considered as the suspected diagnosis.

\section{Mutation Analysis}

Molecular analysis of SLC2A2 gene was performed according to the suspicion of FBS based on the preliminary clinical and laboratory findings. Genomic DNA (Deoxyribonucleic acid) was extracted from peripheral blood leukocyte samples. Sequence analysis was performed by using MiSeq next generation sequencing (NGS) platform, a FDA approved diagnostic system (Illumina, San Diego, CA, USA). Genomic DNA was extracted according to the manufacturer's standard procedure using the QIAamp DNA Blood Midi Kit (Qiagen, Hilden, Germany). All coding exons of the gene and their flanking splice site junctions were amplified using PCR (Polymerase chain reaction) primers, designed with PRIMER $^{\odot}$ Primer Designer v.2.0 (Scientific \& Educational Software programme) software. PCRs were validated by using agarose gel electrophoresis. After PCR amplification, the libraries were prepared with the NexteraXT kit (Illumina Inc.), according to the manufacturer's instructions. NGS was carried out on MiSeq (Illumina Inc.). Sequences were aligned to the hy19 genome within MiSeq Reporter software (Illumina Inc.). Visualization of the data was performed with IGV 2.3 (Broad Institute) software.

\section{Study Design}

Six patients were included into the study for oral glucose tolerance test. Two patients younger than the age of 72 months were excluded because of the possible complications.

To evaluate the glucose tolerance response a standard oral glucose test (OGTT) protocol with $1.75 \mathrm{~g} / \mathrm{kg}$ oral glucose was performed. Fasting and second hour plasma glucose and serum insulin levels were measured respectively. Mean HbA1c levels in the last year were calculated.

\section{Results}

\section{Molecular Analysis}

Patient 1. The diagnosis of FBS was confirmed by DNA analysis showing a novel homozygous NM_000340.1c.1069delGinsAATAA (p. V357̄Nfs*37) mutation of the SLC2A2 gene; both parents were heterozygous carries of this mutation. As this is a frameshift mutation 
causing premature stop codon, this mutation was evaluated as a likely pathogenic variant.

Patient 2-3-4-5-6-7. Sequence analysis was performed for SLC2A2 gene, which revealed a previously reported homozygous mutation, NM_000340.1 c.482_483insC (p. G162Rfs*17) As this is a frameshift mutation causing premature stop codon, this mutation was evaluated as a likely pathogenic variant.

Patient 8. Molecular genetic testing was performed for SLC2A2 gene. A novel homozygous NM_000340.1c.575A > G (p.H192R) mutation was detected and both of the parents were heterozygous for the same mutation. In silico analysis by Mutation taster, SIFT, SIFT Provean and Polyphen 2 softwares predicts this variant as a disease causing variant. This variant is in a conserved region in different species. We screened 1000 exome data done in our center and we did not detect this variant. This variant was evaluated as a likely pathogenic variant.

\section{Oral Glucose Tolerance Test Results}

After an eight-hour fasting period, oral glucose tolerance test with $1.75 \mathrm{~g} / \mathrm{kg}$ oral glucose was performed to the six patients, elder than 3 years old. Significant hypoglycemia was seen only in one patients ( $43 \mathrm{mg} / \mathrm{dl}$ ) (Table II). Following to the oral glucose load, 120 minutes plasma glucose levels were higher than $140 \mathrm{mg} / \mathrm{dl}$. Four of the patients reached the values between $140-199 \mathrm{mg} / \mathrm{dl}$, which could be determined as impaired glucose tolerance. Postprandial 120 minutes plasma glucose levels were higher than $200 \mathrm{mg} / \mathrm{dl}$ in patient 4 and patient 8 , although they did not have diabetes mellitus.

Fasting insulin levels were in the normal range in all of the patients according to the simultaneous plasma glucose levels. 120 minutes peak insulin levels were lower than the normal, except the eighth patient. Mean HbAlc levels were in the normal range. Last HbAlc levels also were between 4.4-6.0, indicating both fasting hypoglycemia and postprandial hyperglycemia.

\section{Discussion}

Fanconi-Bickel Syndrome is a rare inherited disorder of carbohydrate metabolism, caused by the mutations in the facilitative glucose transporter protein 2 (GLUT2/SLC2A2) gene. ${ }^{10}$ GLUT2 provides the glucose release from the hepatocytes during fasting and after feeding GLUT2 transports glucose and galactose into hepatocytes. ${ }^{2}$ Defective GLUT2 transporter protein causes impaired glycogen breakdown and gluconeogenesis and therefore fasting hypoglycemia and postprandial hyperglycemia. ${ }^{5}$ Excessive renal glucose loss also plays an important role in fasting hypoglycemia. ${ }^{11}$

Apart from the facilitative glucose transport, GLUT2 has an important role in insulin secretion from the pancreatic beta cells, which is not clearly defined. ${ }^{11}$ Absence of functional GLUT2 protein causes inadequate insulin secretion. This physiological efflux explains the mechanism of neonatal diabetes as the initial presentation of the SLC2A2 mutations during the course of FBS. ${ }^{12}$ Consistent with the previous studies, impaired glucose tolerance was detected in all of our patients. After an oral glucose load, 120 minutes glucose levels were higher than $140 \mathrm{mg} / \mathrm{dl}$. Beyond that, two patients, patient 4 and patient 8 can be determined as overt diabetes according to the 120 minute glucose levels. However mean $\mathrm{HbAlc}$ levels were in the normal range probably because of the hypoglycemic episodes. Therefore, anti diabetic treatment was not given to these two patients. Frequent followup with oral glucose tolerance tests is planned for these two patients.

While significant fluctuations in blood glucose levels with postprandial hyperglycemia has been reported, phenotypic variability in patients with FBS was a remarkable course even with the same mutations. ${ }^{13}$ As a female infant was presented with diabetic ketoacidosis at the age of 33 days, transient neonatal diabetes is a presentation of FBS. ${ }^{14}$ Beyond these presentations, the considerable changes to the oral glucose load, up to the diabetes mellitus, have only been reported in our cases. It points to the importance of the follow-up with OGTTs among the FBS patients. Furthermore, our cases with significant hyperglycemia notify the need to develop a consensus on the treatment of hyperglycemia and also diabetes.

Although FBS is a rare disease, in the recent studies many mutations have been described in the SLC2A2 gene. ${ }^{5-15}$ Three different 
mutations were detected in 8 patients from six families. Six patients have the NM_000340.1 c.482_483insC (p. G162Rfs*17) mutation, which has been described before, associated with a typical FBS including impaired glucose tolerance. Patient 4 has this mutation and developed diabetic glucose profile at the age of sixteen years old. In this case, the mutation is more moderate and suggests that could lead to the development of diabetes during the follow up.

The novel mutation that is currently described in patient 1; NM_000340.1c.1069delGinsAATAA (p. V357Nfs*37) is a frame shift mutation with a severe presentation including significant rickets and recurrent infections, especially pneumonia. The patient is too young to evaluate the glucose tolerance with an oral glucose tolerance test. Further investigations are needed to predict the course of the disease caused by this mutation.

NM_000340.1c.575A > G (p.H192H) mutation described in patient 8 , is also a novel mutation in the SLC2A2 gene, leading a serious course even in the early stages of the disease. Beyond the impaired glucose tolerance, 120 minutes plasma glucose level pointed out overt diabetes. Even there is a significant increment in postprandial insulin level comparing to the fasting levels, it was not enough to maintain normoglycemia after the oral glucose load.

Consistent with the previous studies, despite their tendency to develop postprandial hyperglycemia, HbAlc levels were in the normal range. ${ }^{16}$ According to these experiences, in order to avoid the risk of severe hypoglycemia, we recommend follow up with conservative treatment and frequent oral glucose tolerance screening.

In conclusion, impaired glucose tolerance is a common finding of FBS related with the causative mutation. Monitoring with oral glucose tolerance tests and $\mathrm{HbA} 1 \mathrm{c}$ levels, even in the younger ones is needed. Requirement for the use of insulin or other medications enhancing the insulin secretion could be decided with the follow up of these patients and further studies.

\section{REFERENCES}

1. Fanconi G, Bickel H. Chronic aminoaciduria (amino acid diabetes or nephrotic-glucosuric dwarfism) in glycogen storage and cystine disease. Helv Paediatr Acta 1949; 4: 359-396.

2. Santer R, Schneppenheim R, Dombrowski A, Götze $\mathrm{H}$, Steinmann B, Schaub J. Mutations in GLUT2, the gene for the liver-type glucose transporter, in patients with Fanconi-Bickel syndrome, Nat Genet 1997; 17: 324-326. Erratum in, Nat Genet 1998; 18: 298.

3. Leturque A, Brot-Laroche E, Le Gall M. GLUT2 mutations, translocation, and receptor function in diet sugar managing. Am J Physiol Endocrinol Metab 2009; 296: 985-992.

4. Santer R, Schneppenheim R, Suter D, Schaub J, Steinmann B. Fanconi-Bickel syndrome the original patient and his natural history, historical steps leading to the primary defect, and a review of the literature. Eur J Pediatr 1998; 157: 783-797.

5. Santer R, Steinmann B, Schaub J. Fanconi-Bickel syndrome a congenital defect of facilitative glucose transport. Curr Mol Med 2002; 2: 213-227.

6. Guillam MT, Hümmler E, Schaerer E, et al. Early diabetes and abnormal postnatal pancreatic islet development in mice lacking Glut-2. Nat Genet 1997; 17: 327-330.

7. Guillam MT, Dupraz P, Thorens B. Glucose uptake, utilization, and signaling in GLUT2-null islets. Diabetes 2000; 49: 1485-1491.

8. Peduto A, Spada M, Alluto A, La Dolcetta M, Ponzone A, Santer R. A novel mutation in the GLUT2 gene in a patient with Fanconi-Bickel syndrome detected by neonatal screening for galactosaemia. J Inherit Metab Dis 2004; 27:279-280.

9. Lee PJ, Van't Hoff WG, Leonard JV. Catch-up growth in Fanconi-Bickel syndrome with uncooked cornstarch. J Inherit Metab Dis 1995; 18: 153-156.

10. Santer R, Groth S, Kinner M, et al. The mutation spectrum of the facilitative glucose transporter gene SLC2A2 (GLUT2) in patients with Fanconi-Bickel syndrome. Hum Genet 2002; 110: 21-29.

11. Thorens B. GLUT2, glucose sensing and glucose homeostasis. Diabetologia 2015; 53: 221-232.

12. Sansbury FH, Flanagan SE, Houghton JA, et al. SLC2A2 mutations can cause neonatal diabetes, suggesting GLUT2 may have a role in human insulin secretion. Diabetologia 2012; 55: 2381-2385.

13. Fridman E, Zeharia A, Markus-Eidlitz T, Haimi Cohen YH. Phenotypic variability in patients with FanconiBickel syndrome with identical mutations. JIMD Rep 2015; 15: 95-104.

14. Setoodeh A, Rabbani A. Transient neonatal diabetes as a presentation of Fanconi-Bickel syndrome. Acta Medica Iranica 2012; 50:836-838. 
15. Temizel IN, Coskun T, Yuce A, Kocak N. Fanconi-Bickel syndrome in three Turkish patients with different homozygous mutations. Turk J Pediatr 2005; 47: 167169.

16. Taha D, Al-Harbi N, Al-Sabban E. Hyperglycemia and hypoinsulinemia in patients with Fanconi-Bickel syndrome. J Pediatr Endocrinol Metab 2008; 21: 581562. 